\title{
Dose-Related Structural Effects of Photodynamic Therapy on Rabbit Choroidal Structure
}

\author{
Wei Du ${ }^{a, b, c, d}$ Yin Chih Lee ${ }^{a, b, c, d}$ Tianfu Wang ${ }^{a, b, c, d}$ Haoran Cui a, b, c, d \\ Hui Xu ${ }^{a, b, c, d}$ Xuan Bao ${ }^{a, b, c, d}$ Xin Tang ${ }^{a, b, c, d}$ Mingwei Zhao ${ }^{\text {a, b, c, d }}$
}

aDepartment of Ophthalmology and Clinical Centre of Optometry, Peking University People's Hospital, Beijing, China; 'beye Diseases and Optometry Institute, Peking University People's Hospital, Beijing, China; 'Beijing Key Laboratory of Diagnosis and Therapy of Retinal and Choroid Diseases, Beijing, China; ${ }^{d}$ College of Optometry, Peking University Health Science Center, Beijing, China

\author{
Keywords \\ Photodynamic therapy - Verteporfin - Dose-dependent \\ effects · Choroidal structure $\cdot$ Central serous \\ chorioretinopathy
}

\begin{abstract}
Introduction: Photodynamic therapy with verteporfin (vPDT) has been shown to be effective against central serous chorioretinopathy (CSC) and was the preferred therapeutic for CSC treatment. However, alterations in choroidal structure after PDT were reported, and these effects were dosedependent. This study aimed to compare the changes in choroidal structure after PDT with different doses of verteporfin in rabbits and may provide individualized therapeutic guidance for patients who failed to respond to initial halfdose vPDT. Methods: The full dose of verteporfin used in CSC was $6 \mathrm{mg} / \mathrm{m}^{2}$, which was used in patients with neovascular age-related macular degeneration. Laser fluence was $50 \mathrm{~J} /$ $\mathrm{cm}^{2}$ (irradiance, $600 \mathrm{~mW} / \mathrm{cm}^{2}, 83 \mathrm{~s}$ ). There were 4 different dose groups in this study $(100 \%, 70 \%, 50 \%$, and $30 \%)$. The alterations were examined at 1 day, 1 week, and 1 month after VPDT using color fundus imaging, indocyanine green angiography, and histopathology analysis. Results: Various de-
\end{abstract}

karger@karger.com www.karger.com/ore

Karger"
(C) 2021 The Author(s)

Published by S. Karger AG, Basel

This is an Open Access article licensed under the Creative Common Attribution-NonCommercial-4.0 International License (CC BY-NC) (http://www.karger.com/Services/OpenAccessLicense), applicable to the online version of the article only. Usage and distribution for commercial purposes requires written permission. grees of choroidal alterations were demonstrated at different dose groups. Examinations on day 1 showed that gradually reduced verteporfin dose tended to decrease photochemical reactions to the choroid in terms of the number of occlusion vessels and area of the lesion. After 1 month, choroid vessel alteration persisted in high-dose groups (100\% and $70 \%)$; nevertheless, alterations of low-dose groups (50\% and $30 \%)$ returned to normal. Conclusions: vPDT can induce photochemical reactions of the choroid, high dose causes permanent change, and low dose causes recoverable change. The dose-dependent alterations need to be considered for the individual therapeutic plan according to the situation of a patient with CSC.

() 2021 The Author(s)
Published by S. Karger AG, Basel

\section{Introduction}

Central serous chorioretinopathy (CSC) is an idiopathic chorioretinopathy that is characterized by serous retinal detachment involving mainly the macular area and accompanied by leakage of altered retinal pigment epithelium (RPE) $[1,2]$. Most acute CSC often resolves spontaneously within a few months; however, in some patients, 
it may progress to chronic CSC, which can cause photoreceptor degeneration and RPE atrophy, resulting in irreversible anatomical and functional damage [3]. Recently, the understanding of the pathology causing CSC changed from the RPE level to the choroidal disturbance level [4, 5]. It was thought that photodynamic therapy (PDT) with verteporfin could alter choroidal vasculature structure and perfusion, reduce subretinal fluid (SRF), and improve visual acuity for both acute and chronic CSC [6-8].

The dose of verteporfin infusion and laser energy parameters used in CSC were identical to those used in neovascular age-related macular degeneration (nAMD) (6 $\mathrm{mg} / \mathrm{m}^{2}$, irradiance, $\left.600 \mathrm{~mW} / \mathrm{cm}^{2}, 83 \mathrm{~s}\right)[9,10]$. It is generally accepted standard therapy regimen in CSC treatment. Although PDT with conventional verteporfin dose yielded favorable effects, post-PDT complications have been reported in patients, such as RPE atrophy, RPE tear, secondary choroidal neovascularization, and choroidal ischemia [11-13]. As a result, many studies on modified PDT protocols have been evaluated, including lowering verteporfin doses, decreasing laser power, decreasing laser treatment times, or shortening the treatment interval [14-17]. Some studies showed that 50\%-dose PDT has favorable results compared with full-dose PDT [14, 18, 19], but there remain failed cases after the initial half-dose PDT. Data on the treatment regimen for patients without response to initial half-dose PDT are insufficient due to the paucity of studies. It has been verified that PDT retreatment for patients with recurrence after failure of initial PDT is effective $[20,21]$. The optimum dose for retreatment should be established; therefore, experimental evidence is needed to clarify the impact of multiple drug applications on tissue construct.

Nevertheless, few animal or clinical studies have compared the photochemical reactions of choroid vessels caused by PDT using different doses of verteporfin. This study aims to evaluate choroid vessel structure changes caused by PDT using different doses of verteporfin contributing to treatment response on choroidal vessels in rabbits. The results of this animal study are crucial for not only determining therapeutic effects but also preventing future complications in individualized CSC treatment.

Fig. 1. Color fundus image. a1 Control group showed normal fundus. a2 Verteporfin-only group. a3 Laser-only group showed normal fundus 1 day after treatment. b1-3 Fundus photographs of the $100 \%$ dose group. c1-3 Fundus photographs of the 70\% dose group. d1-3 Fundus photographs of the 50\% dose group. e1-3 Fundus photographs of the $30 \%$ dose group. Retinal detachment and transient SRF were noted 1 day after treatment in all treatment

\section{Materials and Methods}

\section{Animals}

Adult Chinchilla bastard rabbits (Oryctolagus cuniculus, standard Chinchilla) of either sex weighing 2.5-3.0 kg were used for the experiments (obtained from Beijing Tiantan Biological Products Co., Ltd., Beijing, China). Rabbits were maintained at the Animal Laboratories of the Peking University People's Hospital. Animals were used in accordance with the Association for Research in Vision and Ophthalmology Statement for the Use of Animals in Ophthalmic and Vision Research. All experimental procedures were approved by the Institutional Animal Care and Use Committee of Peking University People's Hospital (permit No. 2014-17).

A total of 33 rabbits were included in this study. They were randomly divided into 7 groups: normal control (3 rabbits), verteporfin only (3 rabbits), laser only (3 rabbits), 100\% dose (6 rabbits), $70 \%$ dose (6 rabbits), 50\% dose (6 rabbits), and $30 \%$ dose (6 rabbits). Rabbits were anesthetized using $3 \%$ isoflurane inhalation via a facemask. Pupils were dilated with $1 \%$ atropine sulfate ophthalmic gel (Xingqi Pharmaceutical Co., Ltd., Shenyang, China), $0.5 \%$ tropicamide, and $0.5 \%$ phenylephrine hydrochloride eye drops (Santen Pharmaceutical Co., Ltd., Osaka, Japan).

After PDT, rabbits were kept under dark conditions for 3 days to prevent light damage. Then, the animals were euthanized with sodium pentobarbital overdose, and the eyes were enucleated for analysis.

\section{PDT Procedures}

The currently accepted standard dose of verteporfin used in $\mathrm{CSC}$ was identical to the dose used in neovascular age-related macular degeneration patients $\left(6 \mathrm{mg} / \mathrm{m}^{2}\right)[9,10]$. Verteporfin (Visudyne, Novartis, AG, Bulach, Switzerland) was used according to the manufacturer's instructions at a concentration of $2 \mathrm{mg} / \mathrm{mL}$ and dissolved in $7 \mathrm{~mL}$ of sterile water; the bottle was protected from light and used within $2 \mathrm{~h}$. The $100 \%$ dose of verteporfin used in rabbits was $0.43 \mathrm{mg} / \mathrm{kg}$ [22], which was extrapolated from the recommended dose in humans of $6 \mathrm{mg} / \mathrm{m}^{2}$. Moreover, $70 \%$ dose was $0.301 \mathrm{mg} /$ $\mathrm{kg}, 50 \%$ dose was $0.215 \mathrm{mg} / \mathrm{kg}$, and $30 \%$ dose was $0.129 \mathrm{mg} / \mathrm{kg}$.

For PDT lesions, a diode laser at $689 \mathrm{~nm}$ with a slit-lamp delivery system (Opal Photoactivator, Lumenis Inc, Santa Clara, CA, USA) was used. Marginal ear vein infusion of verteporfin was performed for $10 \mathrm{~min}$, followed by laser delivery at $15 \mathrm{~min}$ from the start of infusion. The effect of laser illumination was evaluated at a fluence of $50 \mathrm{~J} / \mathrm{cm}^{2}$ (irradiance, $600 \mathrm{~mW} / \mathrm{cm}^{2}, 83 \mathrm{~s}$ ). A factor of 0.66 for the rabbit eye was used [23], and the diameter of the laser spot was $2,500 \mu \mathrm{m}$. Two laser spots were placed at a distance of $1 / 2$ papillary diameter from the inferior optic disc margin. All rabbits received $\mathrm{PDT}$ in 1 eye, and the other eye did not receive any treatment. To ensure consistency, PDT was performed by an experienced ophthalmologist.

groups (b1-e1); 1 week after treatment, the 100\% dose and $70 \%$ dose groups still had retinal detachment and SRF (b2, c2); retinal detachment and SRF disappeared in the 50\% dose and 30\% dose groups (d2, e2); 1 month after treatment, RPE mottling was observed in the $100 \%$ dose and $70 \%$ dose groups $(\mathbf{b} 3, \mathbf{c} 3)$; retinas of the $50 \%$ dose and $30 \%$ dose groups returned to normal (d3, e3). SRF, subretinal fluid.
(For figure see next page.) 


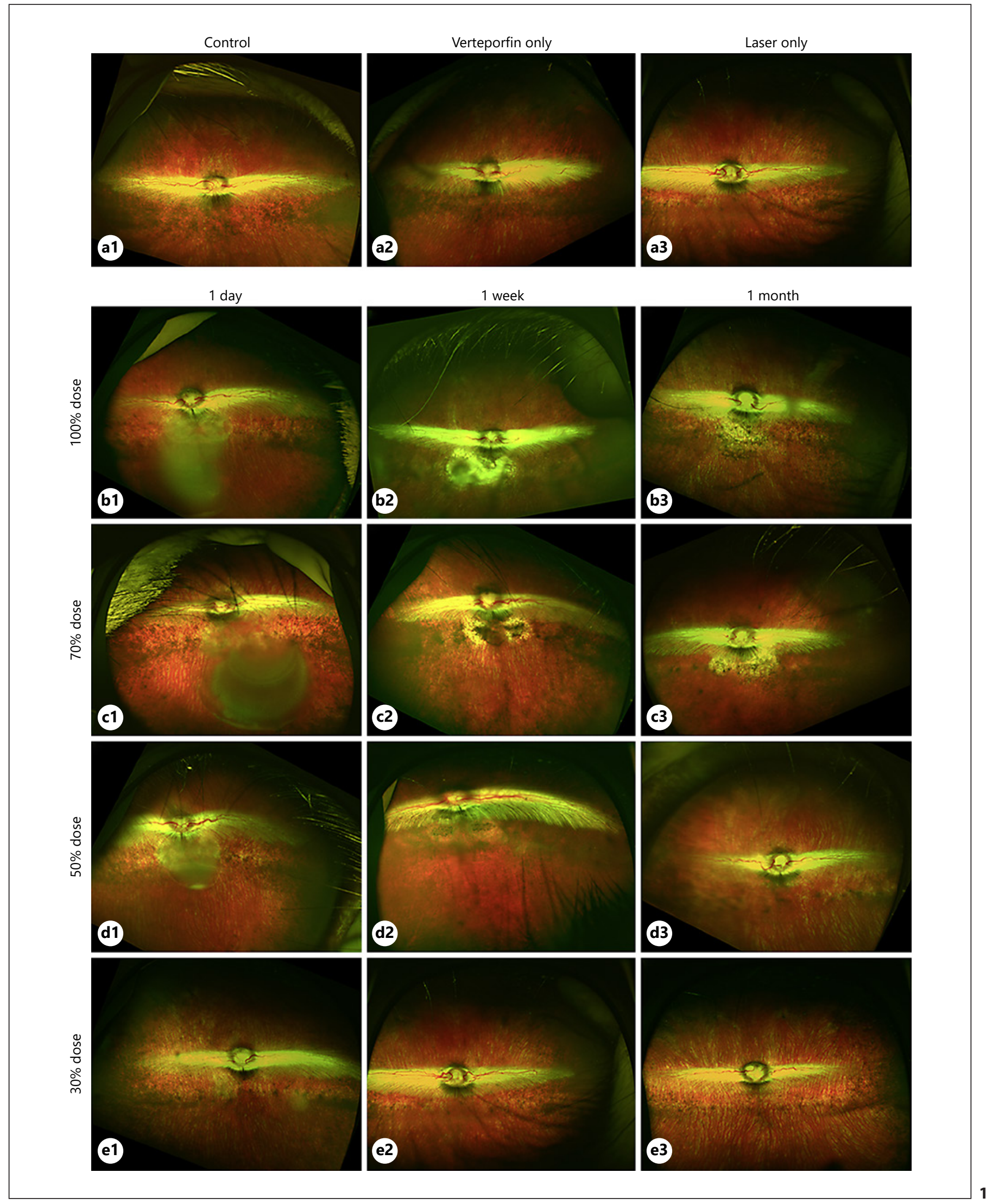


Color Fundus Photography and Indocyanine Green

Angiography

Color fundus photography (Optomap, Optos plc, Scotland, UK) and indocyanine green (ICG) angiography (Heidelberg Tomograph-HRT II, Heidelberg Engineering, Heidelberg, Germany) were performed on both eyes of all rabbits at 1 day $(1 \mathrm{D}), 1$ week $(1 \mathrm{~W})$, and 1 month $(1 \mathrm{M})$ after PDT. The anesthetized animals were injected with $0.5 \mathrm{~mL}$ ICG $(5 \mathrm{mg} / \mathrm{mL}$, Dandong Yichuang Pharmaceutical Co., Ltd., Liaoning, China) via the ear vein. The hyperfluorescent area indicated damage to the choriocapillaris, and hypofluorescent area inside the laser lesion indicated vessel occlusion.

\section{Histopathology}

Eyes were enucleated fixed in 4\% paraformaldehyde overnight at $4^{\circ} \mathrm{C}$ at $1 \mathrm{D}, 1 \mathrm{~W}$, and $1 \mathrm{M}$ after PDT. The anterior segments were removed, and the posterior eyecups were embedded in paraffin, sectioned at $5 \mu \mathrm{m}$, deparaffinized, rehydrated, and stained with hematoxylin and eosin (H-E). Section image analyses were performed using an Olympus microscope (Olympus, Tokyo, Japan).

\section{Electron Microscopy}

The rabbits were sacrificed, and the eyes were collected and fixed in 3\% glutaraldehyde (Electron Microscopy Sciences, Hatfield, PA, USA) at an appropriate time point. Then, the specimens were postfixed with 1\% osmium tetroxide (Electron Microscopy Sciences, PA, USA), dehydrated, and embedded in Epon (Eponate 12, Ted Pella, Redding, CA, USA). The retinal sections were obtained, contrasted with uranyl acetate and lead citrate, and analyzed using a Hitachi transmission electron microscope (Hitachi, Tokyo, Japan).

\section{Results}

\section{Color Fundus Imaging Changes after PDT}

In the verteporfin-only and laser-only groups, the fundus appearance was unchanged $1 \mathrm{D}$ after treatment (Fig. 1a2, a3); however, various degrees of chorioretinal responses were demonstrated in different dose groups. Retinal detachment and transient SRF were gradually decreased from $100 \%$ dose group to $50 \%$ dose group; in $30 \%$ dose group, only slight retinal whitening corresponding to the laser irradiation spot appeared (Fig. 1b1-e1). After $1 \mathrm{~W}$ of treatment, $100 \%$ dose and $70 \%$ dose groups still

Fig. 2. ICG angiography. a1 Control group showed normal ICG angiography image. a2 Verteporfin-only group. a3 Laser-only group exhibited normal choroidal vessel appearance without leakage or occlusion. b1-3 ICG angiography of the 100\% dose group. At 1 day after PDT, hyperfluorescence corresponding to the laser spot size and hypofluorescent patch inside the laser lesion were noted; at 1 week after PDT, hypofluorescence still in the center of the laser spots, with a hyperfluorescent ring in the periphery. At 1 month after PDT, choroid vessels showed reperfusion, but existed in small areas of hypofluorescence; ICG angiography of 70\% dose group (c1- had retinal detachment and SRF, but the detachment area was shrunk, and the SRF was mostly or completely absorbed (Fig. 1b2, c2). Retinal detachment was not observed in 50\% dose group, and pigmentation appeared (Fig. 1d2). In 30\% dose group, whitening of the lesions was barely observed in the treated sites (Fig. 1e2). Retinas of $100 \%$ dose and $70 \%$ dose groups diffused with hyperor hypopigmented patch and RPE mottling were intermingled at $1 \mathrm{M}$ after PDT (Fig. 1b3, c3). Retinas of 50\% dose and 30\% dose groups returned to normal (Fig. 1d3, e3).

\section{ICG Angiography Changes}

One feature common to vPDT response after $24 \mathrm{~h}$ was the presence of a circle of choroidal hyperfluorescence corresponding to the laser spot size and a hypofluorescent patch area inside the laser lesion, suggesting more severe chorioretinal damage and representing vessel occlusion. The higher the verteporfin dose, the larger the hypofluorescent area inside the laser lesion (Fig. 2b1-e1).

Seven days following treatment, gradual reappearance of patent choroidal vessels was observed; however, hypofluorescence was still observed in the center of the laser lesions, with a hyperfluorescent ring in the periphery in the $100 \%$ dose and $70 \%$ dose groups (Fig. 2b2, c2). This pattern remained constant during all phases of the angiography. In the $50 \%$ dose group, persistence of hypofluorescence of the lesion center was observed, while the hyperfluorescence intensity of the surrounding ring markedly decreased (Fig. 2d2). The 30\% dose group did not show any alterations in choroid vessels (Fig. 2e2).

At 30 days after treatment, reperfusion of the occluded choroid vessels occurred in 100\% dose and 70\% dose groups. There were still small areas of hypofluorescence, which demonstrated persistence of perfusion defects in choroid vessels (Fig. 2b3, c3). No angiographic aspect of the choroid vessel was observed in the 50\% dose and 30\% dose groups (Fig. 2d3, e3).

3). Angiography findings were consistent with $100 \%$ dose group. d1-3 ICG angiography of 50\% dose group. At 1 day after PDT, the choroid presented hyperfluorescence ring, and hypofluorescent patch area inside the laser lesion was noted. At 1 week after PDT, hypofluorescence was observed, while the hyperfluorescence ring decreased. At 1 month after PDT, choroid vessels were normal; ICG angiography of the $30 \%$ dose group (e1-3). At 1 day after PDT, angiography findings were consistent with those of the $50 \%$ dose group. At 1 week after PDT, no alteration of choroid vessels was observed. ICG, indocyanine green; PDT, photodynamic therapy.

(For figure see next page.) 


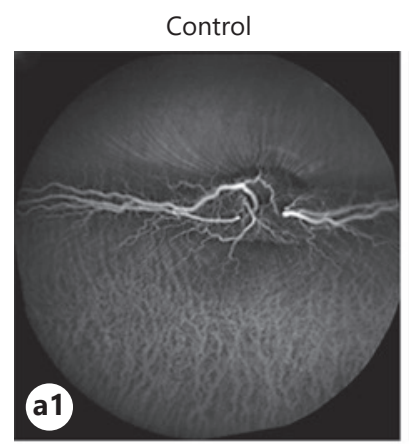

1 day
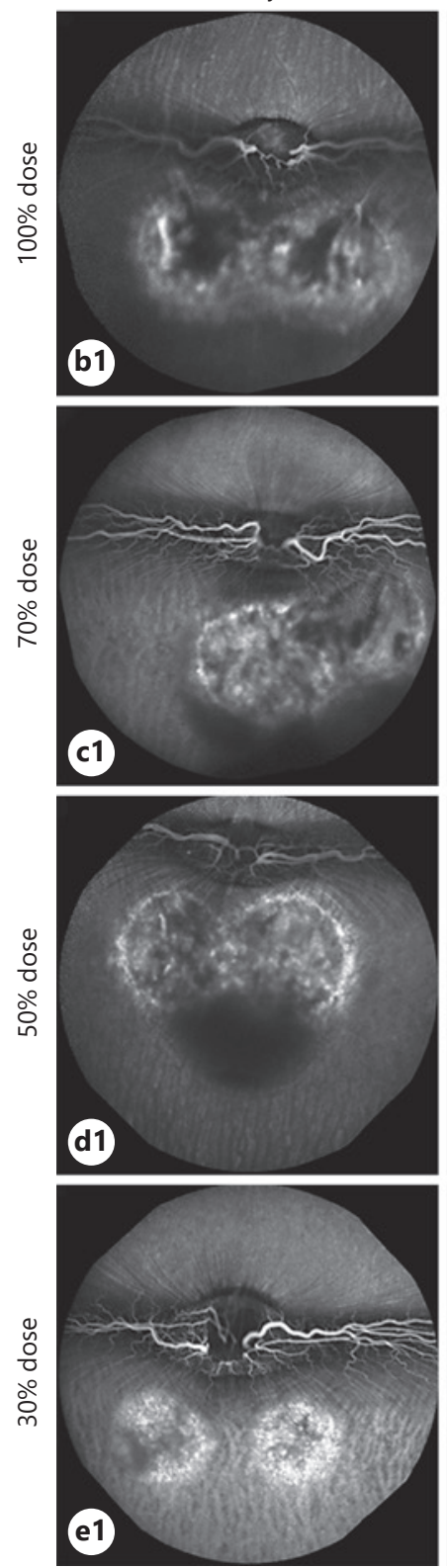

Verteporfin only

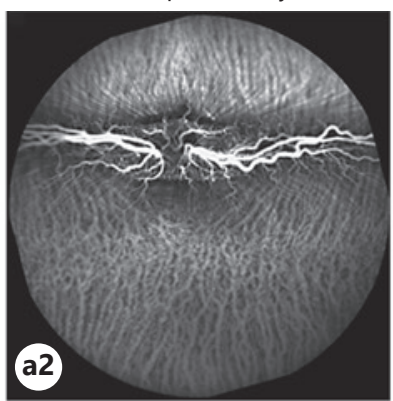

1 week
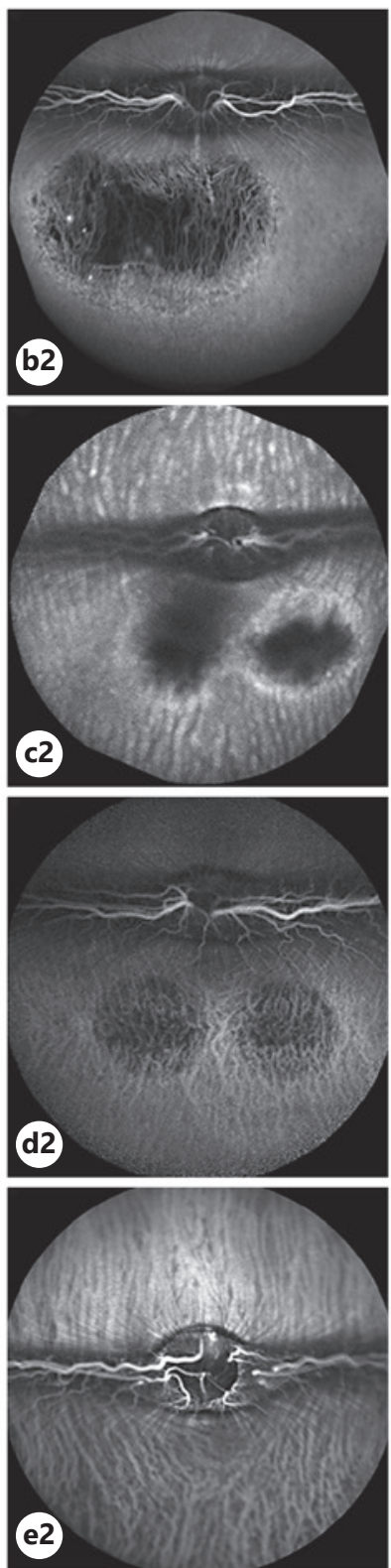

Laser only

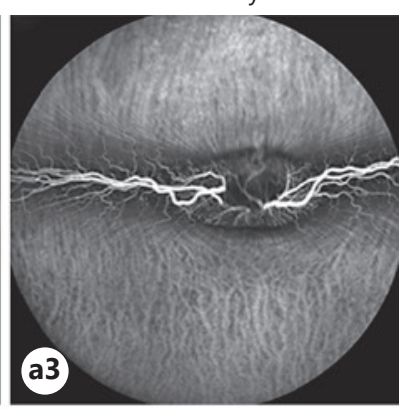

1 month
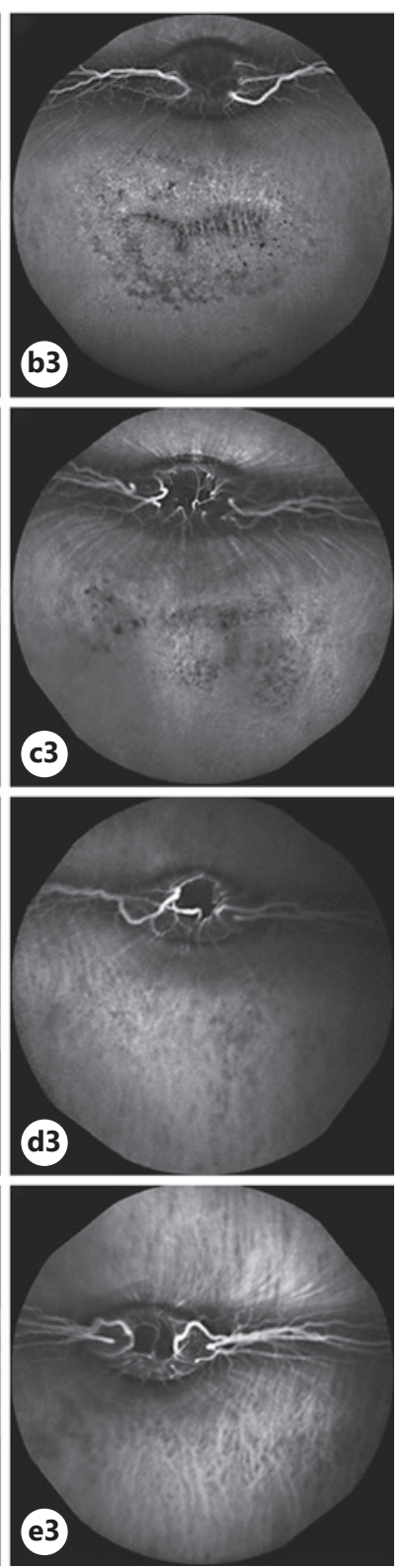


\section{Histopathologic Findings}

Histologic examination of laser-only group and verteporfin-only group showed a normal pattern without any observable pathologic change in the retina and choroid tissue at $1 \mathrm{D}$ after treatment (Fig. 3a-c, Fig. $4 \mathrm{a}-\mathrm{c}$ ). Light microscopy of $\mathrm{H}-\mathrm{E}$ staining and electron microscopy showed a pattern of milder photochemical reaction to the choroid and retina structures with decreasing verteporfin dose levels. The histological changes were mainly the same between the first and second irradiation spots in the same eye. H-E staining sections of $100 \%$ dose and $70 \%$ dose groups revealed not only thrombosis of medium and large size choroidal vessels but also destruction of the RPE and OS (outer segment) and large amount of subretinal exudation $1 \mathrm{D}$ after irradiation. On day 7 after treatment, choroidal vessel thromboses nearly resolved, a small amount of exudation remained, and RPE cell layer discontinuity did not recover. At $1 \mathrm{M}$, the blockage of choroidal vessels almost disappeared, choroid fibrous proliferation and exudation resolved, and there was thinning of the outer nuclear layer (Fig. 3d-e).

In the $50 \%$ dose group, $1 \mathrm{D}$ after PDT, distended choroidal vessels and very few obstructed choriocapillaries can be detected, and modest disruption of RPE, photoreceptor cell layer, and intermediate subretinal exudation were observed. At $1 \mathrm{~W}$ after PDT, dilatation of the choroid vessels was observed, and RPE cell discontinuity did not recover. After $1 \mathrm{M}$, choroid and retina structures returned to normal (Fig. 3f).

Histological damage in the $30 \%$ dose group was milder than that in the $50 \%$ dose group. Small but visible exudation, distended choroidal vessels, and fewer obstructed choriocapillaries were detected in $\mathrm{H}-\mathrm{E}$ staining section on day 1 after PDT. After $1 \mathrm{~W}$, dilatation of the choroid vessels was observed, and other tissues recovered to normal. At $1 \mathrm{M}$, both choroid and retina structure returned to normal (Fig. 3g).

On electron microscopy, intact choriocapillary microstructure was detected in the verteporfin-only and laseronly groups compared with the control group (Fig. 4a-c). All PDT with verteporfin groups showed damage to choriocapillary microstructure $1 \mathrm{D}$ after PDT. Bruch's membrane showed discontinuity, and choriocapillary endothelial cell displayed severe swelling. In the vessel lumens, monocytes and hemolyzed red blood cells were observed (Fig. $4 \mathrm{~d}-\mathrm{g}$ ). The degree of severity differed among the 4 groups. The $100 \%$ dose group (Fig. $4 \mathrm{~d}$ ) and the $70 \%$ dose group (Fig. 4e) had more occluded vessels, and the response degree was milder in the 50\% dose group (Fig. 4f) and the $30 \%$ dose group (Fig. $4 \mathrm{~g}$ ). The $30 \%$ dose group also showed less severe condition than the 50\% dose group. At $1 \mathrm{~W}$ after PDT, the choriocapillary in $100 \%$ dose and $70 \%$ dose groups showed rough basal lamina, and the fibrin was visible in the vascular lumina and the surrounding tissue. The choriocapillary microstructure of $30 \%$ dose and $50 \%$ dose groups almost returned to normal. On $1 \mathrm{M}$ after laser irradiation, abnormalities of the vascular lumen, basal lamina, and endothelial cell were still prominent in 100\% dose group and 70\% dose groups, and the basal lamina was still lightly rough and had occasional discontinuity. The other 2 groups showed a normal vascular microstructure.

\section{Discussion}

PDT was originally used as a clinical therapy for malignant tumors. In 2003, it was proposed for patients with CSC. Previous studies have demonstrated beneficial visual outcomes in most patients $[6,24,25]$. One limitation of the current PDT treatment was the damage to normal structures. Additional adverse effects to the normal retina, RPE, and choroid caused by PDT are dose-dependent $[26,27]$. To prevent adverse effects, half-dose or low-fluence PDT has been studied in the treatment of CSC. A retrospective study conducted by Nicolo et al. [28] revealed that, although half-dose PDT and half-fluence PDT had equal visual improvement and safety in longterm follow-up, half-dose PDT had the advantage of a more rapid reabsorption of the fluid and lower recurrence rate. Decreased fluence PDT might cause more significant inflammatory reaction or increase collateral damage to the normal tissue. To improve treatment safety in CSC, half-dose PDT was proposed. The resolution rates of the SRF after half-dose PDT vary from $60 \%$ to 95\% [29-31]. Our previous study evaluated a range of verteporfin doses from $10 \%$ to $70 \%$ and found that $30 \%$ was the lowest effective dose, while $50 \%$ was better than a $30 \%$ with SRF completely resolved in $95 \%$ of patients [32].

However, recurrence of SRF occurred in some patients (10-20\%) after initial 50\%-dose PDT [33]. These patients required re-treatment. It is believed that patients with persistent SRF for at least 3 months since the previous PDT should benefit from re-treatment, especially if there has been a positive response [34]. To the best of our knowledge, presently, the re-treatment strategy for eyes with persistent SRF did not have uniform standard. The application of higher dose vPDT in patients requiring retreatment leads to a better therapeutic efficacy but may 
Fig. 3. H-E staining of rabbit choroid vessels. a Control group: normal rabbit retina and choroid. b Verteporfin-only group. c Laser-only group showed a normal pattern without any pathologic change. d In the $100 \%$ dose group, at 1 day after PDT, vessel closure of choriocapillaries and deeper choroidal vessels were observable (arrows), OS layer condensed, RPE destructed, and SRF $\left(^{*}\right)$ was damaged. At 1 week after PDT, choriocapillaries thromboses nearly vanished, RPE cell layer showed discontinuity, and a small amount of exudation remained. At 1 month after PDT, choriocapillary occlusion disappeared, and choroid fibrous proliferation and exudation resolved. e In the $70 \%$ dose group, the same was found. $f$ In the $50 \%$ dose group, at 1 day after PDT, distended choroidal vessels, few obstructed choriocapillaries (arrows), and mild SRF $\left.{ }^{*}\right)$ were observed. At 1 week after PDT, dilatation of the choroid vessels was observed, and RPE cells discontinuity did not recover. After 1 month, choroid and retina structure returned to normal. $\mathbf{g}$ The same was found in $30 \%$ dose group, but the damage was milder than that in the $50 \%$ dose group. GCL, ganglion cell layer; IPL, inner plexiform layer; INL, inner nuclear layer; OPL, outer plexiform layer; ONL, outer nuclear layer; OS, outer segment; RPE, retinal pigment epithelium; SRF, subretinal fluid; $\mathrm{H}-\mathrm{E}$, hematoxylin and eosin; PDT, photodynamic therapy; arrow, occluded choroid vessel; *, SRF; scale bar, $50 \mu \mathrm{m}$.

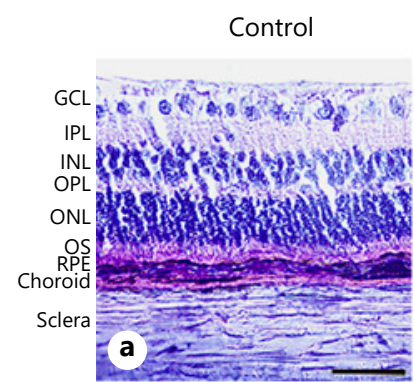

Verteporfin only

Laser only
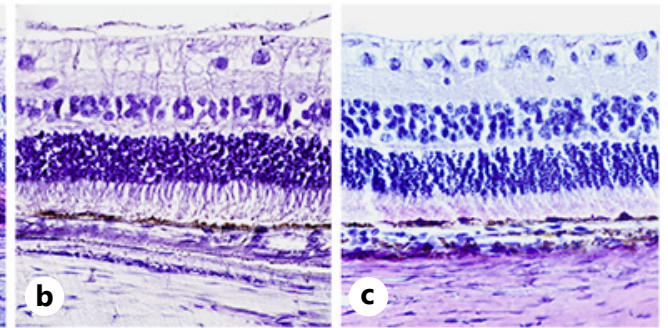

1 day

1 week

1 month
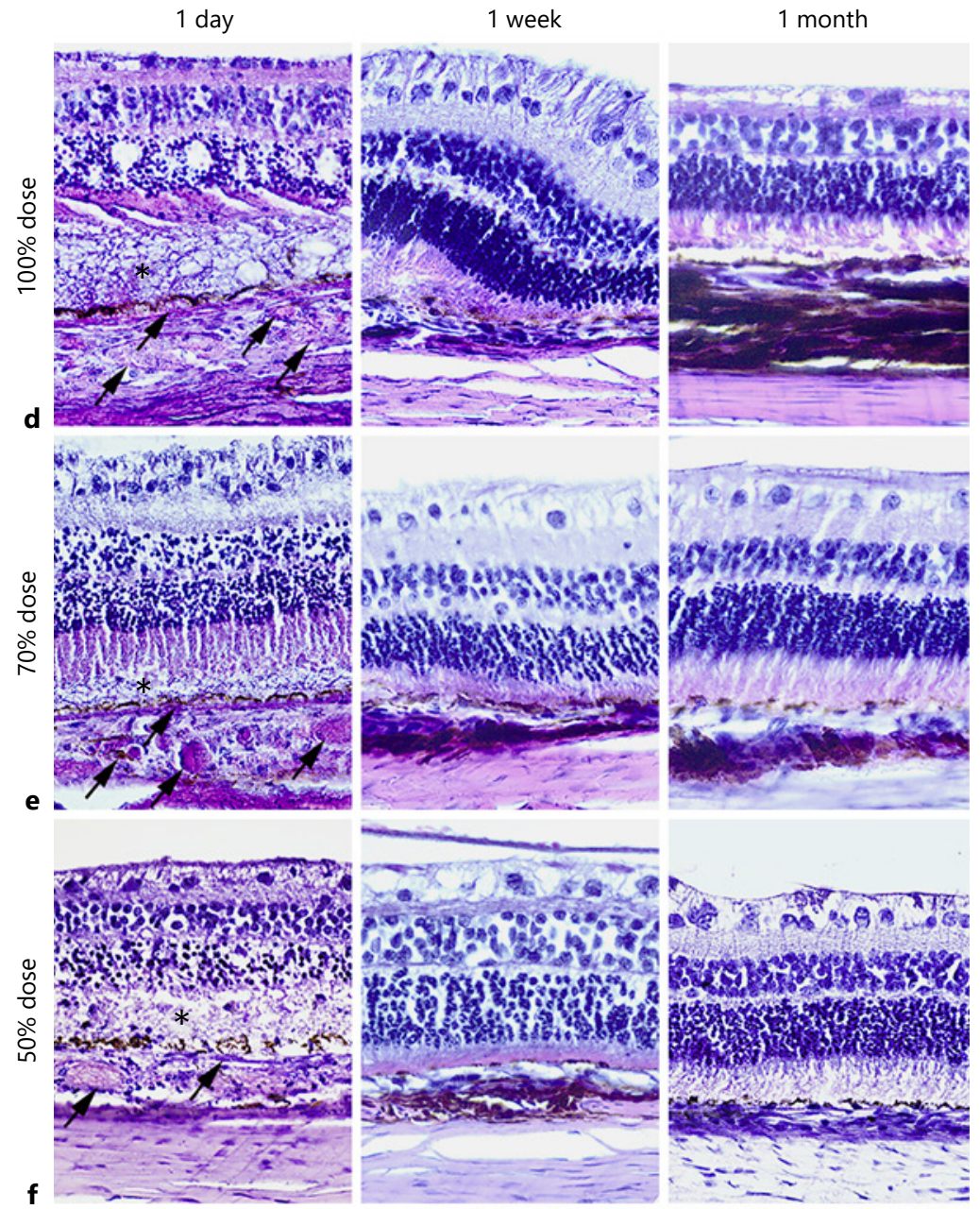

(12)
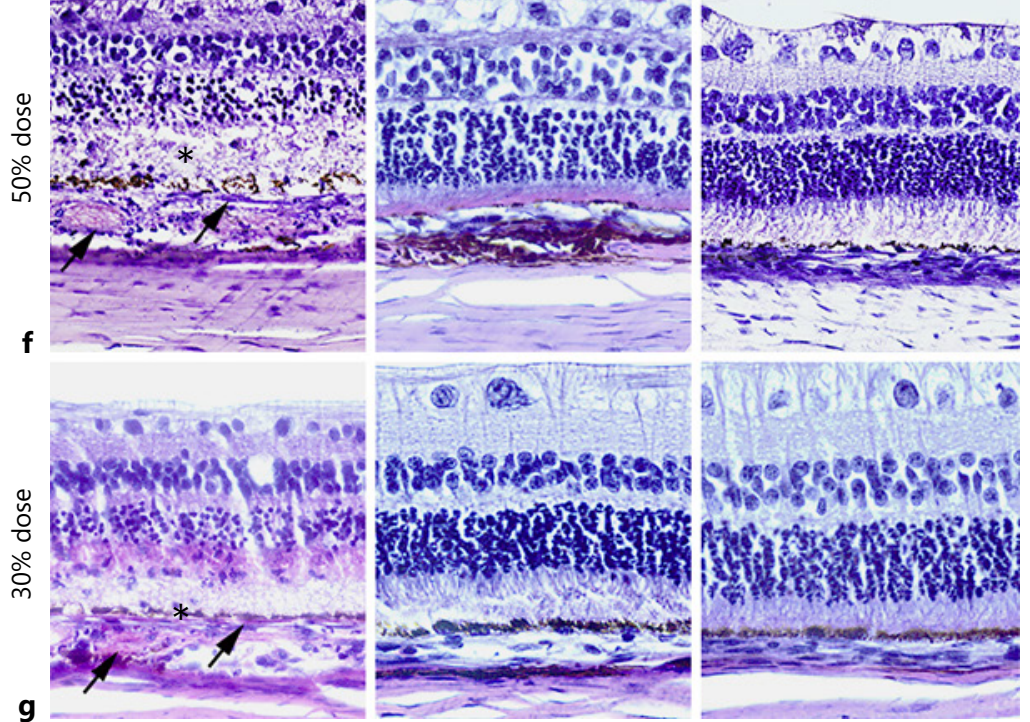

Dose-Related Effects of Photodynamic Therapy on Choroidal Structure
Ophthalmic Res 2021;64:1037-1047

DOI: $10.1159 / 000519328$ 
also result in potential adverse events. It is important to minimize potential retinal toxicity during PDT while maintaining the treatment effects. The complex structural changes of the chorioretinal anatomy that limits in vivo imaging, such as optical coherence tomography and ICG angiography, indicated that much of our understanding of this critical structure has originated from histopathological analysis.

Recently, the new imaging technique of optical coherence tomography angiography (OCTA) has improved the visualization of the chorioretinal vascular structures and microcirculation in vivo [35]. Demircan et al. demonstrated choriocapillaris alterations using OCTA after half-fluence PDT at 3 days and 30 days following therapy. They concluded that there was a markedly decreased choriocapillary flow limited to the site of the PDT spot in the very early period following PDT in eyes with CSC, and the choriocapillary perfusion returned to normal at day 30 [36]. In the study by Alovisi et al. [37] measured by OCTA scan, half-dose PDT seems to produce short-term (1 week after PDT) changes on the luminal component of both the choriocapillary and choroid, which returned to normal status at 1 month from treatment. Although OCTA has proven to be a valuable tool for the depth-resolved evaluation of the retinal and choroidal structures, the structural changes of choriocapillary and choroid tissue after PDT are still unclear.

Normal choriocapillary and choroid structure changes after PDT are unavailable in humans. Our observation represents a novel finding in an animal model. Even 30\%dose vPDT yielded minimal choroid change, as choroidal hyperfluorescence corresponding to the laser spot size and a hypofluorescent patch area inside the laser lesion on fundus photography and ICG angiography were observed. Histopathology showed distended choroidal vessels, obstructed choriocapillaries, and small but visible exudation 1 day after PDT. Choroidal changes aggravated upon increasing verteporfin dose. Until the end of our observational period (1 month after treatment), we continued to observe choroid hypofluorescence and some

Fig. 4. Electron microscopy appearance of rabbit choroid vessels. a Control group. b Verteporfin-only group. c Laser-only group. d $100 \%$ dose group. e $70 \%$ dose group. f $50 \%$ dose group. g $30 \%$ dose group. Intact choriocapillary microstructure was detected in verteporfin-only and laser-only groups compared with that in the control group. At 1 day after PDT, all verteporfin PDT groups showed damage to choriocapillary microstructure. Bruch's membrane showed discontinuity (arrow heads), and choriocapillary endothelial cell displayed severe swelling. In the occluded vessel lumens $\left.{ }^{*}\right)$, cell fragments were observed. At 1 week after PDT, in the $100 \%$ pigment mottling in the RPE in high-dose groups $(70 \%$ dose and $100 \%$ dose) on fundus photography and ICG angiography, but low-dose groups (30\% dose and 50\% dose) recovered to normal. Previous clinical studies showed that PDT works by producing temporary and reversible choriocapillary hypoperfusion or occlusion. Our findings are in agreement with these observations. We also confirmed that photochemical reactions in PDT on the choriocapillary and choroid are also dose-dependent.

It is well known that cytotoxicity in PDT mainly results from the production of high reactive singlet oxygen $\left({ }^{1} \mathrm{O} 2\right)$ or oxygen radical ions, which is generated from the reaction of photosensitizer promoted to an excited state under light [38]. Oxygen radicals specifically act on vascular endothelial cell and lead to vascular endothelial cell alteration. Consequently, subretinal exudation was detected after treatment. Under different levels of verteporfin dose, the degrees of choriocapillary alteration are different. Therefore, it might be speculated that with low verteporfin dose, the effect of PDT is just small endothelium lesions (angiographic hyperfluorescence without vessel occlusion) rather than vessel clotting, so the initiation of a $50 \%$ dose might be too low to have a significant effect in some patients. With increasing verteporfin dose, the alteration in choroid vessel increased, may further promote vascular occlusion or thrombosis (angiographic hypofluorescence), and plays a positive role in patients requiring re-treatment. However, it may potentially cause side effects when the verteporfin dose was extremely high.

A human clinical trial reported full-dose vPDT, revealed choroidal hypoperfusion with choriocapillary destruction at 2 years of observation [39], and suggested permanent closure of part of choroidal vasculature. Some clinical studies confirmed the $30 \%$ dose seemed to be safe and effective in CSC treatment; therefore, successful rate in the $30 \%$ dose was < that in the $50 \%$ dose, and recurrence rate in the $30 \%$ dose group was $>$ the $50 \%$ dose group $[32,40,41]$. Thus, in our clinical work, patients with failure to initial half-dose treatment required retreatment with the same dose or gradually increased

dose and 70\% dose groups choriocapillary showed rough basal lamina, and cell debris was visible in the vessel lumen. Choriocapillary microstructure of the $30 \%$ dose and $50 \%$ dose groups almost recovers to normal. At 1 month after PDT, abnormalities of vascular lumen and endothelial cell were still prominent in the $100 \%$ dose and 70\% dose groups. Moreover, the 50\% dose and 30\% dose groups appeared a normal vascular microstructure. En, endothelial cell; Pe, pericyte; RPE, retinal pigment epithelium; OS, outer segment; PDT, photodynamic therapy; arrowhead, Bruch's membrane; *, vessel lumen; scale bar, $2 \mu \mathrm{m}$.

(For figure see next page.) 


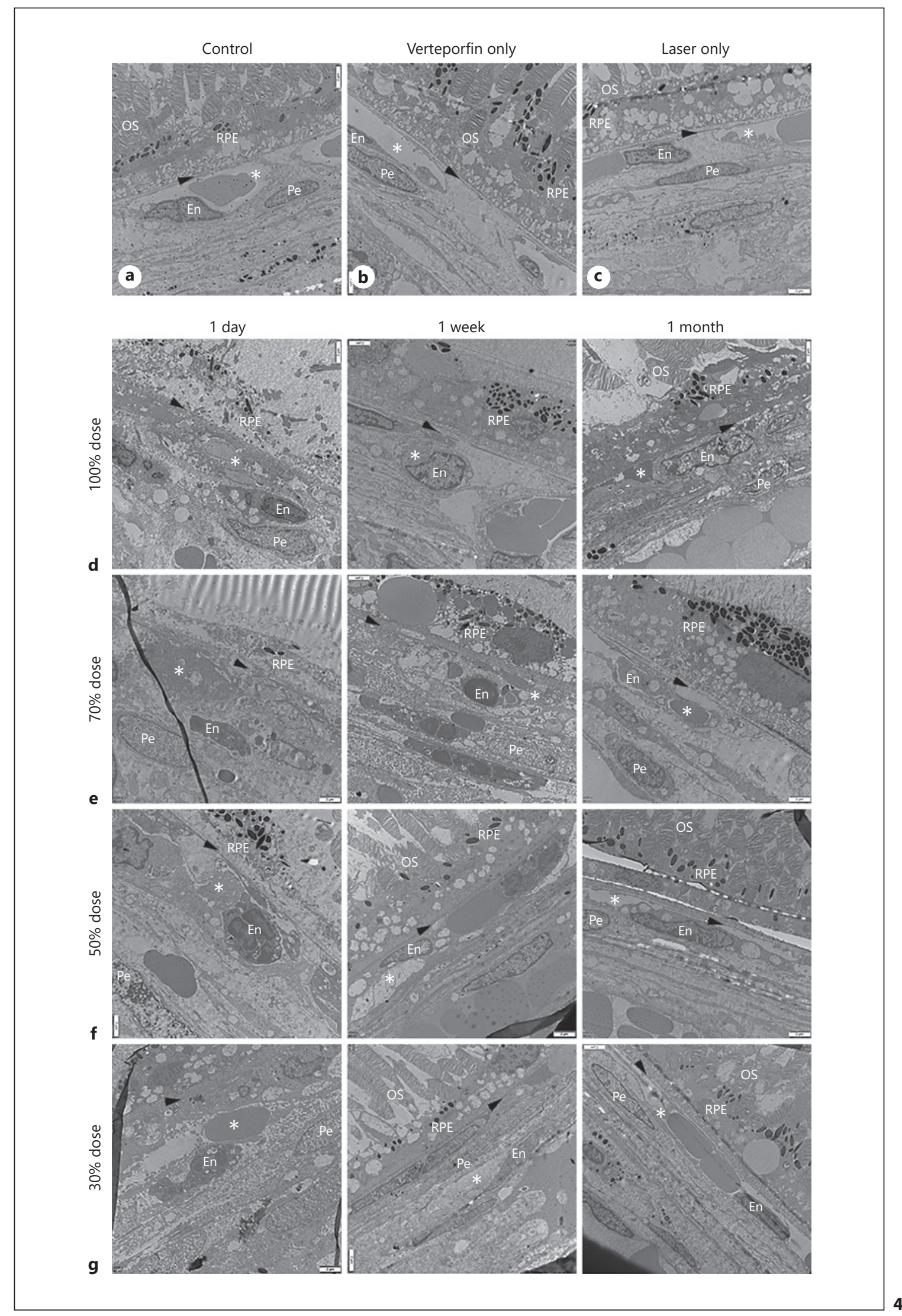


verteporfin dose. In the retrospective study of $\mathrm{Oh}$ et al. [42], patients with relapse were received additional treatment, all had a good clinical prognosis with a decrease in or a complete absorption of SRF, and none of them had ocular or systemic adverse events. The dose of re-treatment was not reported in their study. However, through histological experiments, we showed that more serious tissue damage occurred in the higher dose group. Observations of the PDT effect on the choroid tissue are valuable for treatment selection in clinical practice. Eyes with persistent SRF or recurrence after initial half-dose PDT, they needed additional re-treatment. It is crucial for patients to select the appropriate re-treatment option. Therefore, we advocate starting with low dose and escalating to full dose based on treatment response in clinical PDT.

Our study has several limitations, including small sample size and short-term follow-up period. To avoid more sacrifice, we used 6 rabbits for each group. Moreover, the adverse effect of PDT on choroidoretinal function should be observed after treatment of different doses.

In summary, PDT with verteporfin used clinically in the treatment of CSC induces injury of the physiological choroid, high dose may cause permanent alteration, and low dose causes slight change. The ophthalmologist should make individualized treatment plans according to patients' situation, especially for those patients who failed in the initial PDT and required multiple re-treatments. This will be beneficial for patients to reach optimal therapeutic effect and diminish side effects.

\section{Acknowledgments}

We thank Ms. Yanan Hu and Qi Luo for technical support in electron microscopy.

\section{Statement of Ethics}

All applicable international, national, and/or institutional guidelines for the care and use of animals were followed. All experimental procedures performed in studies involving animals were in accordance with the ethical standards of Institutional Animal Care and Use Committee of Peking University People's Hospital (permit No. 2014 - 17).

\section{Conflict of Interest Statement}

The authors have no conflicts of interest to declare.

\section{Funding Sources}

This study was funded by the National Natural Science Foundation of China (No. 81600770); National Key R\&D Program of China (2020YFC2008203); Beijing Municipal Natural Science Foundation, China (No. 7164306); and Research Fund for Science and Technology Program of Beijing (Z161100000516037).

\section{Author Contributions}

Wei Du wrote this manuscript. Mingwei Zhao provided the idea of this study, performed PDT, and revised this manuscript. Wei Du, Yin Chih Lee, Tianfu Wang, Haoran Cui, Hui Xu, Xuan Bao, and Xin Tang performed the experiment process. All the authors have read and approved the final manuscript.

\section{Data Availability Statement}

All data generated during this study are included in this article. Further enquiries can be directed to the corresponding author.

\section{References}

1 Daruich A, Matet A, Dirani A, Bousquet E, Zhao M, Farman N, et al. Central serous chorioretinopathy: recent findings and new physiopathology hypothesis. Prog Retin Eye Res. 2015 Sep;48:82-118.

2 Vogel RN, Langlo CS, Scoles D, Carroll J, Weinberg DV, Kim JE. High-resolution imaging of intraretinal structures in active and resolved central serous chorioretinopathy. Invest Ophthalmol Vis Sci. 2017 Jan 1;58(1): 42-9.

3 Levine R, Brucker AJ, Robinson F. Long-term follow-up of idiopathic central serous chorioretinopathy by fluorescein angiography. Ophthalmology. 1989 Jun;96(6):854-9.
4 Piccolino FC, Borgia L, Zinicola E, Zingirian $M$. Indocyanine green angiographic findings in central serous chorioretinopathy. Eye. 1995;9 (Pt 3)(Pt 3):324-32.

5 Prunte C, Flammer J. Choroidal capillary and venous congestion in central serous chorioretinopathy. Am J Ophthalmol. 1996 Jan; 121(1):26-34

6 Cardillo Piccolino F, Eandi CM, Ventre L, Rigault de la Longrais RC, Grignolo FM. Photodynamic therapy for chronic central serous chorioretinopathy. Retina. 2003 Dec;23(6):752-63.

7 Chan WM, Lam DS, Lai TY, Tam BS, Liu DT, Chan CK. Choroidal vascular remodelling in central serous chorioretinopathy after indo- cyanine green guided photodynamic therapy with verteporfin: a novel treatment at the primary disease level. Br J Ophthalmol. 2003 Dec;87(12):1453-8.

8 Ober MD, Yannuzzi LA, Do DV, Spaide RF, Bressler NM, Jampol LM, et al. Photodynamic therapy for focal retinal pigment epithelial leaks secondary to central serous chorioretinopathy. Ophthalmology. 2005 Dec;112(12):2088-94.

9 Toa-rmdwptTS Group. Photodynamic therapy of subfoveal choroidal neovascularization in age-related macular degeneration with verteporfin: one-year results of 2 randomized clinical trials--TAP report 1 . Arch Ophthalmol. 1999 Oct;117(10):1329-45. 
10 Verteporfin In Photodynamic Therapy Study G. Verteporfin therapy of subfoveal choroidal neovascularization in age-related macular degeneration: two-year results of a randomized clinical trial including lesions with occult with no classic choroidal neovascularization-verteporfin in photodynamic therapy report 2. Am J Ophthalmol. 2001 May;131(5):54160.

11 Colucciello M. Choroidal neovascularization complicating photodynamic therapy for central serous retinopathy. Retina. 2006 Feb; 26(2):239-42.

12 Isola V, Pece A, Parodi MB. Choroidal ischemia after photodynamic therapy with verteporfin for choroidal neovascularization. Am J Ophthalmol. 2006 Oct;142(4):680-3.

13 Lee PY, Kim KS, Lee WK. Severe choroidal ischemia following photodynamic therapy for pigment epithelial detachment and chronic central serous chorioretinopathy. Jpn J Ophthalmol. 2009 Jan;53(1):52-6.

14 Chan WM, Lai TY, Lai RY, Liu DT, Lam DS Half-dose verteporfin photodynamic therapy for acute central serous chorioretinopathy: one-year results of a randomized controlled trial. Ophthalmology. 2008 Oct;115(10): 1756-65.

15 Reibaldi M, Boscia F, Avitabile T, Russo A, Cannemi V, Uva MG, et al. Low-fluence photodynamic therapy in longstanding chronic central serous chorioretinopathy with foveal and gravitational atrophy. Eur J Ophthalmol. 2009 Jan-Feb;19(1):154-8.

16 Reibaldi M, Boscia F, Avitabile T, Uva MG, Russo A, Zagari M, et al. Functional retinal changes measured by microperimetry in standard-fluence vs low-fluence photodynamic therapy in chronic central serous chorioretinopathy. Am J Ophthalmol. 2011 Jun;151(6): 953-60.

17 Rouvas A, Stavrakas P, Theodossiadis PG, Stamatiou P, Milia M, Giannakaki E, et al Long-term results of half-fluence photodynamic therapy for chronic central serous chorioretinopathy. Eur J Ophthalmol. 2012 MayJun;22(3):417-22.

18 Fujita K, Yuzawa M, Mori R. Retinal sensitivity after photodynamic therapy with half-dose verteporfin for chronic central serous chorioretinopathy: short-term results. Retina. 2011 Apr;31(4):772-8.

19 Shinojima A, Kawamura A, Mori R, Fujita K, Yuzawa M. Detection of morphologic alterations by spectral-domain optical coherence tomography before and after half-dose verteporfin photodynamic therapy in chronic central serous chorioretinopathy. Retina. 2011 Oct;31(9):1912-20.
20 Park YJ, Kim YK, Park KH, Woo SJ. Longterm efficacy and safety of photodynamic therapy in patients with chronic central serous chorioretinopathy. Ophthalmic Surg Lasers Imaging Retina. 2019 Dec 1;50(12):76070.

21 Mohabati D, Boon CJF, Yzer S. Risk of recurrence and transition to chronic disease in acute central serous chorioretinopathy. Clin Ophthalmol. 2020;14:1165-75.

22 Administration UFaD. Guidance for industry: estimating the maximum safe starting dose in initial clinical trials for therapeutics in adult healthy volunteers. 2005

23 Birngruber R, Gabel VP, Hillenkamp F. Experimental studies of laser thermal retinal injury. Health Phys. 1983 May;44(5):519-31.

24 Taban M, Boyer DS, Thomas EL, Taban M. Chronic central serous chorioretinopathy: photodynamic therapy. Am J Ophthalmol. 2004 Jun;137(6):1073-80.

25 Yannuzzi LA, Slakter JS, Gross NE, Spaide RF, Costa DL, Huang SJ, et al. Indocyanine green angiography-guided photodynamic therapy for treatment of chronic central serous chorioretinopathy: a pilot study. Retina. 20032012 Feb;32(Suppl 1):288-98.

26 Schlotzer-Schrehardt U, Viestenz A, Naumann GO, Laqua H, Michels S, Schmidt-Erfurth U. Dose-related structural effects of photodynamic therapy on choroidal and retinal structures of human eyes. Graefes Arch Clin Exp Ophthalmol. 2002 Sep;240(9):74857.

27 Framme C, Flucke B, Birngruber R. Comparison of reduced and standard light application in photodynamic therapy of the eye in two rabbit models. Graefes Arch Clin Exp Ophthalmol. 2006 Jul;244(7):773-81.

28 Nicolo M, Eandi CM, Alovisi C, Grignolo FM, Traverso CE, Musetti D, et al. Half-fluence versus half-dose photodynamic therapy in chronic central serous chorioretinopathy. Am J Ophthalmol. 2014 May;157(5):1033-7.

29 Lai TY, Chan WM, Li H, Lai RY, Liu DT, Lam DS. Safety enhanced photodynamic therapy with half dose verteporfin for chronic central serous chorioretinopathy: a short term pilot study. Br J Ophthalmol. 2006 Jul;90(7):86974.

30 Chan WM, Lai TY, Lai RY, Tang EW, Liu DT, Lam DS. Safety enhanced photodynamic therapy for chronic central serous chorioretinopathy: one-year results of a prospective study. Retina. 2008 Jan;28(1):85-93.
31 Altinel MG, Kanra AY, Totuk OMG, Ardagil A, Kabadayi K. Comparison of half-dose versus half-fluence versus standard photodynamic therapy in chronic central serous chorioretinopathy. Photodiagnosis Photodyn Ther. 2020 Nov 3:102081.

32 Zhao M, Zhang F, Chen Y, Dai H, Qu J, Dong C, et al. A $50 \%$ vs $30 \%$ dose of verteporfin (photodynamic therapy) for acute central serous chorioretinopathy: one-year results of a randomized clinical trial. JAMA Ophthalmol. 2015 Mar;133(3):333-40.

33 van Rijssen TJ, van Dijk EHC, Yzer S, OhnoMatsui K, Keunen JEE, Schlingemann RO, et al. Central serous chorioretinopathy: towards an evidence-based treatment guideline. Prog Retin Eye Res. 2019 Nov;73:100770.

34 Fine HF, Ober MD, Hariprasad SM. Current concepts in managing central serous chorioretinopathy. Ophthalmic Surg Lasers Imaging Retina. 2014 Jan-Feb;45(1):9-13.

35 Jia Y, Tan O, Tokayer J, Potsaid B, Wang Y, Liu JJ, et al. Split-spectrum amplitude-decorrelation angiography with optical coherence tomography. Opt Express. 2012 Feb 13;20(4): 4710-25.

36 Demircan A, Yesilkaya C, Alkin Z. Early choriocapillaris changes after half-fluence photodynamic therapy in chronic central serous chorioretinopathy evaluated by optical coherence tomography angiography: preliminary results. Photodiagnosis Photodyn Ther. 2018 Mar;21:375-8.

37 Alovisi C, Piccolino FC, Nassisi M, Eandi CM Choroidal structure after half-dose photodynamic therapy in chronic central serous chorioretinopathy. J Clin Med. 2020 Aug 24;9(9): 2734.

38 Sitnik TM, Henderson BW. The effect of fluence rate on tumor and normal tissue responses to photodynamic therapy. Photochem Photobiol. 1998 Apr;67(4):462-6.

39 Schmidt-Erfurth UM, Michels S. Changes in confocal indocyanine green angiography through two years after photodynamic therapy with verteporfin. Ophthalmology. 2003 Jul;110(7):1306-14.

40 Zhao MW, Zhou P, Xiao HX, Lv YS, Li CA, Liu GD, et al. Photodynamic therapy for acute central serous chorioretinopathy: the safe effective lowest dose of verteporfin. Retina. 2009 Sep;29(8):1155-61.

41 Uetani R, Ito Y, Oiwa K, Ishikawa K, Terasaki $\mathrm{H}$. Half-dose vs one-third-dose photodynamic therapy for chronic central serous chorioretinopathy. Eye. 2012 May;26(5):640-9.

$42 \mathrm{Oh} \mathrm{BL}, \mathrm{Yu}$ HG. Choroidal thickness after fullfluence and half-fluence photodynamic therapy in chronic central serous chorioretinopathy. Retina. 2015 Aug;35(8):1555-60.
Dose-Related Effects of Photodynamic

Therapy on Choroidal Structure
Ophthalmic Res 2021;64:1037-1047

DOI: $10.1159 / 000519328$ 